\title{
A life course approach to cardiovascular aging
}

\author{
Rebecca Hardy', Debbie A Lawlor² \& Diana Kuh*,1
}

\begin{abstract}
A life course approach in epidemiology investigates the biological, behavioral and social pathways that link physical and social exposures and experiences during gestation, childhood, adolescence and adult life, and across generations, to later-life health and disease risk. We illustrate how a life course approach has been applied to cardiovascular disease, highlighting the evidence in support of the early origins of disease risk. We summarize how trajectories of cardiometabolic risk factors change over the life course and suggest that understanding underlying 'normal' or 'healthy' trajectories and the characteristics that drive deviations from such trajectories offer the potential for early prevention and for identifying means of preventing future disease.
\end{abstract}

Coronary heart disease (CHD) and stroke are rare until middle age, but the pathophysiological process of atherosclerosis, which ultimately leads to cardiovascular disease (CVD), is initiated in early life. Fatty streaks and clinically significant raised lesions have been observed in autopsy studies of 15-19-year-old individuals [1] and fatty streaks have even been found to occur in the aorta of premature fetuses, particularly among those of mothers with hypercholesterolemia during pregnancy [2]. Hence, it is important to understand the factors from across life that may influence the development and progression of the disease process. Recent guidelines have emphasized evidence that long-term and cumulative exposure to modifiable risk factors from an early age advances the disease process. The calculation of lifetime as well as short-term (10-year) risk of CVD, particularly for men and women in midlife with a low short-term risk, has been recommended by the American College of Cardiology/American Heart Association [3] and the Joint British Societies [4], while the International Atherosclerosis Society recommends risk assessment be based on lifetime risk in the management of dyslipidemia [5]. A life course approach to cardiovascular (CV) health is further promoted by the American Heart Association, who have provided metrics for children and adults in relation to health behaviors (smoking, physical activity, diet and BMI) and health factors (diabetes, total cholesterol and blood pressure [BP]) [6]. We suggest that life course epidemiological research has shown that all these CV metrics have their origins in early life through a multiplicity of pathways, and here provide some evidence relating to cardiometabolic function (specifically BP, lipid profiles and markers of diabetes, such as glucose and insulin); the early origins of health behaviors are reviewed elsewhere $[7,8]$.

To date, there is a greater body of evidence relating preadult influences with cardiometabolic measures or disease outcomes at a single time point in adulthood, with less attention paid to the factors that drive changes in cardiometabolic function. In this article, after an introduction to life course epidemiology, we describe the trajectories of cardiometabolic function across the whole of

'MRC Unit for Lifelong Health \& Ageing at UCL, 33 Bedford Place, London, WC1B 5JU, UK

${ }^{2} \mathrm{MRC}$ Integrative Epidemiology Unit at the University of Bristol, Oakfield House, Oakfield Grove, Bristol, BS8 2BN, UK

*Author for correspondence: rebecca.hardy@ucl.ac.uk

\section{KEYWORDS}

- adiposity $\bullet$ birth

weight $\bullet$ blood pressure

- cardiovascular disease

- cohort studies - glucose

- life course $\bullet$ lipids

- longitudinal modeling

- social inequalities 
life. We highlight the evidence linking selected life course risk factors to CVD and these cardiometabolic trajectories, and review evidence suggesting that changes in cardiometabolic risk factors at particular periods of the life course may be associated with disease onset.

Life course epidemiology

A life course approach in epidemiology investigates the biological, behavioral and social pathways that link physical and social exposures and experiences during gestation, childhood, adolescence and adult life, and across generations, to later-life health and disease risk $[9,10]$. Adult chronic disease, particularly cardiometabolic and respiratory disease, was the initial focus of life course epidemiology, although this approach has been extended subsequently to other health outcomes. The ground-breaking studies of Barker and colleagues in the 1980s that linked birth weight and other measurements of birth size (as proxy markers of growth in utero) to cardiometabolic disease in later life [11] were a catalyst for the development of life course epidemiology. The programming or 'Barker hypothesis', which states that exposures during critical (or sensitive) developmental periods have long-term consequences for chronic disease, evolved into the more general developmental origins of the adult disease model that includes the impact of postnatal as well as prenatal development on health. The role of early-life socioeconomic conditions has also been widely investigated in relation to CVD, and although initially seen as a competing model to the fetal programming hypothesis, life course epidemiology emphasized the importance of both early biological and social factors.

Prior to the re-emergence of life course ideas in epidemiology in the 1990s [12], the adult lifestyle and risk factor model of health had been dominant, as it had been successful in identifying many modifiable causes of disease and in particular CVD, such as hypertension and inactivity. Life course epidemiology acknowledges the importance of these adult risk factors, but it adds a life course perspective by recognizing their tracking from childhood to adulthood. In terms of health-related behaviors, it emphasized the need to understand the predictors of the initiation and cessation of health-promoting and health-damaging behaviors across life. The life course perspective thus integrated and extended these three apparently conflicting theories of disease etiology: fetal programming, social causation and adult lifestyle. Life course epidemiology attempts to understand the relative importance of exposures from across the whole of life, their interactions and the underlying processes.

The initial concepts of life course epidemiology were proposed by Ben-Shlomo and Kuh as a theoretical basis for the testing of life course hypotheses [10]. Initially, two broad types of life course model - a critical period model and an accumulation of risk model - were proposed, with each type having several submodels (see Ben-Shlomo and Kuh [10] for a detailed review). The difficulties in practice of empirically teasing out the particular life course models have been highlighted [13,14]. More generally, the statistical models used for the testing of lifecourse hypotheses are complex due to repeated and correlated outcome and exposure measures and the methods are still developing and evolving $[15,16]$. Whatever the statistical approach, an understanding of the underlying biology and careful consideration of confounding variables and other potential biases are required. The influence of chance events and processes must also be recognized [17]. Such random processes mean that any life course model is unable to predict individual outcomes and can only provide information on how the development of risk varies across groups.

More recently, life course epidemiology has emphasized the importance of age-related changes in functional capability, using tests of strength and physical performance (e.g., grip strength, standing balance and gait speed) and cognitive performance [18]. Life course epidemiology is thus interested in modeling developmental trajectories that may provide clues to the early-life exposures influencing development, as well as the age-related decline in functioning that occurs postmaturity [18]. The rate of functional decline reflects growing impaired physiological responses to environmental and behavioral challenges. In relation to CVD, understanding how markers of cardiometabolic function change across life and identifying underlying 'normal' or 'healthy' trajectories and the factors associated with deviation in these trajectories are potentially important for understanding the development of CVD and for preventing future disease [19].

Other recent developments focus on how life course epidemiology can go beyond simply demonstrating associations to determining 
whether associations are causal. Replication of results in several cohort studies, in particular in studies from populations with contrasting confounding structures, increases confidence that the observed associations may be causal. Another approach is the use of instrumental variables, with genetic instrumental variables being most commonly used to date, with the technique being termed 'Mendelian randomization' [20]. Because the genetic instrumental variable is related to the outcome only through the exposure and is unrelated to any confounders of exposure and outcome, the relationships between instrument and outcome and between instrument and exposure can be used to derive an unconfounded estimate of the causal effect of exposure on outcome.

Life course trajectories of cardiometabolic function

Longitudinal studies in which repeated measures of a cardiometabolic risk factor are recorded from the same individuals over time are required to investigate true within-individual age-related change. Trajectories inferred from cross-sectional studies can be influenced by secular or birth cohort differences. Of all the cardiometabolic risk factors, life time trajectories in $\mathrm{BP}$ have been by far the most extensively studied [19].

\section{- Life course trajectories of BP}

There is still no single study with BP measured in the same individuals across the whole of life. Hence, an alternative to examining life course trajectories in a single cohort, and an improvement over cross-sectional analyses of individuals of different ages, is to compare multiple longitudinal cohorts with repeated measurements that cover different periods of life. One study modeled systolic BP (SBP) data from seven UK prospective cohort studies, each with at least two measurements of BP and each covering different but overlapping periods of the life course from 7 to $>80$ years of age [21]. Four life course phases were observed: a rapid increase in SBP coinciding with peak adolescent growth; a more gentle increase in early adulthood; a midlife acceleration beginning in the fourth decade; and a deceleration in late adulthood in which increases in SBP slowed and at very old age appeared to decline. A greater increase in SBP in boys compared with girls was observed in childhood, and by the mid-20s, SBP was markedly higher in men compared with women. By contrast, a greater midlife increase was observed in women compared with men, so that by approximately 60 years of age, mean SBP was higher in women. Other cross-sectional and longitudinal studies generally support these findings, with SBP increasing monotonically across most of adult life. Diastolic BP (DBP) has been observed to increase to the fifth decade, and thereafter to plateau or decline $[22,23]$. The midlife rate of increase in DBP, similarly to that of SBP, is greater in women compared with men, but is not as steep as that for SBP [23].

Project HeartBeat! is a detailed study of BP in childhood [24] in which BP was assessed repeatedly every 4 months for 4 years in approximately 700 children initially aged 8,11 or 14 years. Mean levels of SBP increased steeply between ages 8 and 16 years and then leveled off, with the rate of increase being greater in males compared with females. There were no clear ethnic differences. For phase $4 \mathrm{DBP}$, there was a curvilinear association, with the rate of increase reducing after approximately 13 years, whereas the rate of age-related increases for phase 5 was more constant. Mean phase 4 DBP was higher in males than females at all ages, and both measures of DBP were higher in black children at all ages. However, rates of change were similar in males and females and in blacks and non-blacks.

At the other end of life in the seventh and eigth decades, the extent to which the slowing of the increase, or even decrease, in BP seen in most studies is driven by survivor bias (i.e., survival of those who are most healthy, with lower BP and less prone to premature mortality) or effective treatment for lowering BP remains unclear. The decline has been less evident in analyses that have excluded individuals on antihypertensive medication [21], suggesting reduction of BP by treatment may play a role. However, age-related weight loss, arterial stiffening and changes in the autonomic control of BP are possible agerelated processes that could explain a decline [25].

There is evidence of considerable variation in the extent to which BP rises druing adulthood. An occupational cohort (the Whitehall II study) had a mean SBP trajectory that did not begin the accelerated rise until a later age when compared with the general population cohorts in the UK cross-cohort study [21]. A comparative study observed little age-related change in BP from the four nonindustrialized remote populations that were considered, which was in contrast to the 
substantial increases seen in western populations [26]. A recent longitudinal study of adult lowland forager horticulturalists from Bolivia found that BP did increase with age, particularly among women, but that the rate of increase was considerably less than that in the general US population [27]. As well as differences between populations, there is likely to be considerable variation within populations regarding the average trajectories, which are not evident when simply modeling the means. In a latent class analysis of midlife British adults aged 36-53 years, three subgroups of BP change were identified [28]. In both men and women, and for both SBP and DBP, there was a large 'normal' group, which included over $90 \%$ of participants and was characterized by steady increases. There was also a smaller group in which the rate of increase was three- to fourtimes greater than that of the large 'normal' group, and in women, there was a third subgroup in whom BP was consistently high. A subsequent US study in men and women aged 18-30 years at baseline was able to identify a group of individuals whose BP remained low over the course of the study, along with four other groups with increasing or relatively high BP [29]. Identifying such apparently healthy groups and their charactersitics may help to identify novel life course interventions. Overall, these findings demonstrating considerable variation both within and between populations provide no clear evidence that marked midlife increases in BP are part of a natural physiological aging process, but instead may suggest that western lifestyles are driving the increases.

- Life course trajectories of lipids \& glucose Early-life lipid changes vary between boys and girls [30,31]. In a prospective study, levels of total cholesterol and LCL-cholesterol (LDLC) declined with age from 8 to 16 years, with this decline being more rapid in boys than in girls in a prospective study, while triglyceride levels increased with increasing age more markedly in boys than girls [31]. Patterns of change in HDL-cholesterol (HDL-C) were very different between girls and boys, with a sinusoidal pattern in boys with a peak at approximately 11 years of age and a trough at approximately 15 years of age, but little change in levels in girls until 15 years of age, when levels increased. No ethnic differences in mean levels or change in total cholesterol were found, but the mean levels of LDL-C, HDL-C and triglycerides at all ages were more adverse in non-black compared with black children [31].

There are only a few longitudinal studies of age-related changes in lipids in adults. In western industrialized countries, total cholesterol, LDL-C and triglycerides increase from early adulthood to at least the sixth decade of life, and HDL-C declines over this same period $[32,33]$. Total cholesterol, LDL-C and triglyceride levels are greater in men in early adulthood, but the slope of increase from the early 30 s to later life is greater in women than in men, and similarly, HDL-C is lower in men in early adulthood, but the decline over adulthood is greater in women $[32,33]$. In more recent studies, the magnitude of the increase with age was lower, which may reflect greater use of statins and/or improvements in lifestyle [32]. Data from repeat US NHANES cross-sectional surveys suggest that mean fasting glucose in males and females and all ethnic groups remain constant from age 12-20 years [34]. Fasting and postload glucose levels have been found to increase with age from early adulthood to old age, with a suggestion of a sharp increase in the rate of increase in glucose and insulin from approximately 60 years of age [35]. Gender differences appear less marked than those seen for SBP and total cholesterol. This adult age-related change is probably driven by age-related weight gains and declines in physical activity [36].

\section{Life course biological transitions}

As well as the long-term changes, there are also biological transitions in which particularly rapid changes in cardiometabolic trajectories occur, such as puberty and, in women, during pregnancy and the menopause transition. Mean trends in risk factors with age may be too crude to identify effects of the changes relating to such events, particularly as they occur at different ages in different individuals. Whether the patterns of change during such transitions are related to subsequent cardiometabolic trajectories and subsequent disease development remains unclear, even though the timing of such reproductive transitions have long been investigated in relation to CV health in women. Acknowledging the metabolic changes that occur during pregnancy, pregnancy has been proposed as a physiological 'stress test' that reveals latent chronic disease [37], while more generally, reproductive health has been suggested as a 'sentinel of chronic disease' [38]. 


\section{- Puberty}

Some, but not all studies show an association between earlier age at menarche in girls and subsequent increased CHD risk [39] and worse CV risk factors [40-42], but the extent to which this is explained by the higher adiposity in adulthood and/or the tracking of BMI from childhood remains unclear, as few studies have later-life measures of body size.

\section{- Pregnancy}

Detrimental changes to the CHD risk profile, including a relative degree of insulin resistance and hypertriglyceridemia, which are necessary for fetal growth and development, occur during pregnancy, but how long they remain after delivery is not known. Associations between earlier age at first pregnancy and larger numbers of pregnancies with higher rates of CVD may be due to confounding by preparenthood factors and be mediated by health-related behaviors rather than the biological effects of pregnancy. Studies that have compared the association between number of children and CVD and $\mathrm{CV}$ risk factors in men and women can help to disentangle the pathways, but to date, such studies are few and their results are not entirely consistent $[43,44]$.

In addition to the metabolic changes in a normal pregnancy, many of the factors that contribute to CVD, such as inflammation, vasculopathy, angiogenesis, thrombosis, insulin resistance and endocrine profiles, also underlie aberrant menstrual patterns, female infertility, pregnancy complications and adverse pregnancy outcomes [38]. In particular, four common pregnancy complications - gestational diabetes, hypertensive disorders, low birth weight and preterm birth - may reveal CV maternal risks. Women experiencing pre-eclampsia during pregnancy have a higher rate of subsequent hypertension [45] and CHD and stroke [46] than women who have normotensive pregnancies. Offspring birth weight predicts maternal lifespan and CV mortality, with lower birth weight being associated with increased risk [47], and mothers who deliver a preterm baby also have an increased risk of CHD $[48,49]$. The combination of preterm birth, low birth weight for gestational age and pre-eclampsia appear to be additive; one study, for example, found that women with all three characteristics had a risk of CHD admission or death that was seven-times greater than for women with none of these characteristics [48].

\section{- Reproductive aging}

Earlier menopause has been associated with an increased risk of CHD [50], and as noted previously, both SBP and DBP increase more in midlife in women than they do in men. However, prospective studies that have modeled $\mathrm{BP}$ as women go through the menopause suggest little or no specific effect of the menopause per se, over and above that due to aging [51]. By contrast, it has been suggested that there was a specific increase in total cholesterol and LDL-C at approximately the final menstrual period, with the same level being maintained 6 years later [51]. The prevailing view that the estrogen drop during the menopausal transition triggers somatic aging, probably through detrimental changes to risk factor levels, is being challenged. Ovarian aging may be a result of somatic aging or underlying disease or their common antecedents. A study based on the Framingham cohort linked premenopausal high BP and poor lipid profile with early menopause [52]. This led the authors to propose that, rather than being a result of menopause or being linked by common factors, detrimental changes in CHD risk factors may determine age at menopause.

A few studies have shown that women who suffer from vasomotor symptoms (hot flashes and night sweats) during the menopause have an increased risk of subsequent CHD [53], subclinical CVD [54] and a poorer CV risk factor profile [55]. The design of many of the existing studies, however, again means that it is not possible to determine whether CHD risk factors and vascular changes precede vasomotor symptoms, whether symptoms are a marker of underlying vascular changes or whether the two have common antecedents.

\section{Life course risk factors for adult cardiometabolic function \& CVD: examples}

We now provide a brief overview of the evidence relating the commonly studied life course risk factors of prenatal and postnatal body size and socioeconomic position (SEP) to CVD development. Having summarized the evidence in relation to disease end points, we then concentrate on the associations of these factors with the continuous measures of cardiometabolic risk factors, and where possible, highlight research on factors influencing the shape of the life course trajectories. Other early-life factors, such as diet, physical activity and psychological stress, are 
not reviewed here, but may play a role in CVD development, either as independent risk factors or mediators of the associations between body size or SEP and cardiometabolic function.

\section{- Developmental origins of CVD}

Since the original work by Barker, multiple studies have investigated associations between birth weight and later-life outcomes, with many being the subject of systematic reviews and meta-analyses. One systematic review has shown a consistent association of lower birth weight with increasing rates of $\mathrm{CHD}$ [56]. The association between birth weight and diabetes may be U-shaped in populations with high levels of maternal obesity and gestational diabetes [57], and it is possible that this may, in future, translate into increases in CHD at the high end of birth weight as well. A life course approach encouraged investigation of whether the associations between early-life growth and adult disease were confounded by socioeconomic circumstances, as had initially been suggested. Generally, it was found that there was little evidence of such confounding or of consistent modification by later-life factors.

Low birth weight is associated with higher BP in adulthood, but only weakly associated with adverse lipid profiles in childhood and adulthood [58,59]. It has been suggested that the negative association between birthweight and SBP, although initiated in utero, is amplified with age [60]. The initial evidence in support of this hypothesis came from comparisons of multiple studies with BP measured at different ages [60], and so the apparent amplification of the association might actually be a result of stronger associations in historical compared with more contemporary birth cohorts. The suggestion of amplification within individuals is equivalent to hypothesizing that those of lower birth weight have faster increases in BP than others. Studies with repeated measures of BP within the same sample in adulthood suggest that the association between birthweight with BP remains constant at all adult ages [61,62], and two studies have found no amplification of the effect between childhood and early adulthood [63,64], but one study suggested an increase in the effect between adolescence and early adulthood [65]. These discrepancies could be due to differences in whether current body size was adjusted for [66].

Birth weight has been the most commonly used, although not the only marker of fetal development, primarily because it is widely available in historical studies. However, birth weight is a combination of gestational age and fetal growth rate at different periods of pregnancy. Contemporary cohorts that have initiated follow-up in pregnancy have more sophisticated measures; for example, the Southampton Women's Study recorded ultrasound measurements in pregnancy to enable the calculation of fetal growth in early, mid and late pregnancy [67], but such cohorts are still young. Older cohorts do not have adequate variation in gestational age to assess the potentially independent effects of fetal growth and gestational age, but with more premature babies ( $<37$ weeks gestation) surviving into adulthood in younger cohorts, understanding the implications of prematurity on later CHD risk is an increasingly important priority. Younger gestational age has so far been related to higher BP through to midlife [68]. There are also increasing numbers of babies being conceived through assisted reproductive technolgies (ART) and being delivered by cesarean section. A metaanalysis of 13 studies suggested that children delivered by cesarean section had greater odds of obesity than those delivered naturally [69], but there is as-yet little information regarding the relationship with other cardiometabolic factors. The fact that babies conceived through ART have, on average, lower birth weights and greater risks of preterm birth than those conceived naturally suggests that they may be at increased risk of subsequent CHD. There is currently some evidence suggesting increased $\mathrm{BP}$ and vascular dysfunction risk in children who were conceived through ART, although more research is required to replicate such findings in more recent cohorts given the rapid improvement in assisted reproduction techniques, as well as to tease out the mechanisms that are involved [70].

\section{- Childhood \& adolescent body size \& CVD}

A number of aspects of postnatal body size, such as infant growth, tempo of height growth, pubertal growth and childhood obesity, may be important in shaping later CVD risk. Reviews of the existing literature suggest that high BMI in late childhood/adolescence is associated with a higher risk of CHD, but there is less and somewhat conflicting evidence in relation to BMI in infancy/early childhood [71-73]. A large Danish cohort with repeated measures of BMI found that the positive associations between BMI and $\mathrm{CHD}$ were strengthened with the older age of BMI measurement from 7 to 13 years [73]. The 
Helsinki Birth Cohort Study observed that those who developed CHD were smaller than others in infancy, but showed more rapid increases in BMI up to 12 years of age [74,75]. Thus, the strength and direction of association may change with age at BMI measurement. From a public health perspective, it is important to establish whether becoming normal weight in adult life reverses any adverse effects of childhood overweight, but few existing studies have been able to adjust for adult body size. One study observed only slight attenuation of the association of adolescent overweight with $\mathrm{CHD}$ upon the addition of adult BMI, although there were only 38 events and thus confidence intervals were wide [76].

Greater adiposity at any time of life is crosssectionally associated with higher BP, and greater childhood adiposity is associated with higher adult BP. In studies with repeat measurements of indicators of adiposity, those who lose weight have BP levels that are, on average, similar to those who started with a healthier weight $[77,78]$. However, since both BMI and BP track from childhood to adulthood, it is hard to disentangle the length of overweight from any impact of current size [79]. Cohort effects might also be present, as secular increases in overweight and obesity have been accompanied by decreases in BP, raising the possibility that the strength of association between BMI and BP may vary by cohort [8]. As for BP, greater adiposity at any age is associated with more adverse lipids, glucose and insulin, and there is evidence that weight loss, including in childhood, results in improvements in lipid profiles [77]. An analysis of four cohorts followed from childhood to early midlife found that individuals who had been overweight or obese in childhood but were not obese as adults had risks of hypertension, abnormal lipid profiles and increased carotid intimamedia thickness (cIMT) similar to those who had normal BMI consistently from childhood to adulthood [80]. Further analysis in one of these cohorts - the Cardiovascular Risk in Young Finns study - found a higher cumulative lifetime BMI score based on four measures from childhood through to early adulthood was associated with higher cIMT and higher SBP and glucose levels. Mendelian randomization using the FTO gene as the instrumental variable representing lifetime exposure to BMI supported a causal relationship between high cumulative BMI and these same outcomes [81]. A study using a larger and older sample and a genetic score comprising three single-nucleotide polymorphisms known to have the largest effects on BMI suggested a causal association with IHD [82]. However, such studies cannot assess the relative importance of childhood and adolescent BMI compared with adult BMI.

As well as early-life adiposity, linear growth has also been studied, since shorter adult height is associated with higher rates of CHD [83]. Adult height, and in particular adult leg length, can be considered as a biomarker of early-life environmental factors reflecting prepubertal growth, having been related more strongly than trunk length to breastfeeding and higher-energy diets in early childhood and a more advantaged earlylife socioeconomic environment [84,85]. Greater adult height and leg length, but less so greater trunk length, have generally been related to lower BP, better lipid profiles and lower glucose [86-90]. In the Medical Research Council (MRC) National Survey of Health and Development (NSHD), shorter leg length was not only associated with BP level, but also with faster agerelated increases in BP and pulse pressure during midlife [91], thus suggesting that detrimental early-life influences on vascular structure and function may increase vulnerability to the effects of aging on the arterial tree. In the same study, it was also demonstrated that the relationship between a better lipid profile in adulthood and longer adult leg length was reflected in similar associations with taller height at 2 years of age [88], and a more recent study now suggests that short height in childhood is associated with adverse cIMT levels in early old age [92].

\section{- Life course social inequalities}

The socioeconomic inequalities in health within the western Industrialized countries of the world are well documented. SEP in childhood, as indicated by the father's occupational social class, education, income or living conditions, has been shown to be associated with CHD in a multitude of studies [93,94] over and above the influence of education and adult SEP. The mechanisms underlying many of the life course SEP differences in health remain to be elucidated, but are likely to include behavioral and psychosocial pathways, although several studies highlighted the importance of education as a mediator [95].

A systematic review found consistent evidence that those from the most adverse SEP in adulthood had the highest mean BP and a 
greater risk of hypertension, [96], but generally, no association has been observed between childhood SEP (i.e., parental SEP) and BP measured in childhood, adolescence or early adulthood [97]. By contrast, a number of cohort studies have found that those from a more disadvanatged childhood SEP have higher BP in adulthood, independent of adult SEP $[62,98]$. In the MRC NSHD, with repeat measurements of adult BP, there was evidence of an amplification of the adverse effect of low childhood SEP on adult BP [62]. Such amplification with age could explain why little or no association is found between childhood SEP and BP measured in childhood and early adulthood, but that an association between childhood SEP and adult BP has been found. Disadvantaged conditions in childhood have also been linked to more adverse lipid and glucose profiles in adulthood [99-101], although there is some variation in the findings, with own education being more important in some studies [102]. In a study attempting to disentangle the types of life course SEP models of cardiometabolic risk factors, the lifetime accumulation model was predominant in women and the childhood sensitive period model was predominant in men [101].

Linking cardiometabolic trajectories to disease progression \& outcomes

$\mathrm{BP}$ in adolescence/early adulthood is related to $\mathrm{CHD}$ and stroke mortality in follow-ups of cohorts of alumni and military conscripts $[103,104]$. In the Cardiovascular Risk in Young Finns study, SBP assessed between 12 and 18 years of age was positively associated with cIMT assessed at 33-39 years of age, with a magnitude similar to that of the association with SBP in adulthood assessed at the same time as cIMT [105]. For LDL-C, the earlier measure was more strongly related to cIMT [105]. In a subsequent multicohort study, including the Cardiovascular Risk in Young Finns study as well as the Childhood Determinants of Adult Health Study, the Bogalusa Heart Study and the Muscatine Study, which investigated the importance of age at measurement of the risk factor, higher total cholesterol from 12 years of age and higher SBP from 6 years of age were associated with higher young adult cIMT [106]. Associations with these risk factors measured at younger ages (3 years of age for SBP and 3, 6 and 9 years of age for cholesterol) were weak or null, as were the associations between triglyceride levels measured at all ages from 3 to 18 years of age and cIMT. In the Bogalusa Heart Study, the cumulative burden of high SBP (as indicated by the area under the SBP curve) over a 23-year period of childhood and early adult life, as well as a measure of SBP in adolescence/early adulthood, was associated with left ventricular mass index [107]. Evidence in support of a causal association between CHD and cumulative SBP exposure comes from a Mendelian randomization study suggesting that single-nucleotide polymorphisms associated with lower SBP were related to a slower age-related rises in SBP and a considerably greater reductions in risk of CHD than has been found in observational studies [108].

Evidence is accumulating to suggest that the midlife rises in BP have implications for subsequent disease development. The Lifetime Risk Pooling Project found that participants who were consistently hypertensive over at least 10 years and those whose BP increased to the level of hypertension had particularly high cumulative lifetime risks of CHD, while those whose BP decreased to normal levels had an estimated risk similar to those with BPs that had always been in the normal range [109]. In the CARDIA study, in which five latent classes of BP change were defined, groups with trajectories that were elevated-stable and elevated-increasing had higher odds of having a high coronary artery calcification score than the comparison low-stable group [29]. In a UK study, adults belonging to the subgroup with more marked increases in midlife SBP and DBP were more likely to have undiagnosed angina according to the Rose questionnaire than other participants [28]. In the same study, greater midlife increases in SBP were associated with poorer cardiac structure, including left ventricular function at 60-64 years of age [110]. Greater increases between 43 and 53 years of age were shown to be a stronger risk factor of higher left ventricular mass index than more recent BP increases between 53 and 60-64 years of age. Whether this finding represents a detrimental impact of the rate of increase during a particular sensitive period of adult life or whether there is a lag effect that is independent of age or is a result of reverse causality remains to be seen. Whatever the mechanism, the implication for practice is that the possibility of identifying those who are at risk of future disease according to their rate of change in $\mathrm{BP}$, rather than through a single measurement (with a threshold for hypertension), should be further investigated. 


\section{Conclusion}

Evidence from this article shows that changes in $\mathrm{CV}$ and metabolic function start early in life, long before the manifestation of disease, and that the rate of change varies by age and at times of biological transitions, most notably during pregnancy. There is strong evidence that early-life risk factors are involved in the initiation of adverse functional change. Despite cohort studies of adults and children collecting repeated measures of cardiometabolic risk factors, few have fully utilized these data and explored the influences driving change in the measures or how change is related to disease progression. Understanding how markers of cardiometabolic function change across life and the characteristics associated with deviation from what might be considered underlying 'normal' age-related change is key to fully understanding the life course epidemiology of these conditions, and for identifying means of preventing future disease.

\section{Future perspective}

We envisage a continued development of the life course approach in CVD prevention, as highlighted in current guidelines, with increased recognition of the importance of changes in risk factors throughout life, including during pregnancy in women. The initiation of preventative measures to improve lifestyle at all stages of life in order to prevent CVD in later life is

\section{EXECUTIVE SUMMARY}

\section{Life course epidemiology}

- Life course epidemiology provides a framework by which to study cardiovascular aging across the life course.

\section{Life course trajectories of cardiometabolic function}

- Repeated measures of cardiometabolic risk factors in the same individuals across large periods of the life course are preferable to comparisons of cross-sectional data, which are subject to secular and cohort effects.

- Four life course phases for systolic blood pressure have been identified: a rapid increase coinciding with peak adolescent growth; a more gentle increase in early adulthood; a midlife acceleration; and a deceleration or decline in late adulthood.

- Longitudinal data on lipids and glucose trajectories are limited.

\section{Life course transitions}

- Puberty, pregnancy and the menopause transition are times of rapid change in cardiometabolic risk factors.

- Women's reproductive health can be seen as a sentinel of chronic disease, and there is evidence that problems in pregnancy unmask underlying cardiovascular disease (CVD) risk.

\section{Life course risk factors for CVD}

- There is a large body of evidence suggesting that the origins of CVD are in early life and in utero.

- Prenatal and postnatal growth are associated with later CVD and cardiometabolic risk trajectories, but the precise mechanisms behind this are not yet fully understood.

- Social inequalities in CVD risk are initiated in childhood.

\section{Linking cardiometabolic trajectories to disease progression \& outcomes}

- Evidence suggests that childhood measures of cardiometabolic health are at least as strongly associated with cardiovascular outcomes as adult measures and that cumulative exposure may be important.

- Changes in blood pressure in midlife are increasingly being found to be associated with CVD progression and outcomes.

\section{Conclusion}

- Understanding how markers of cardiometabolic function change across life and the characteristics associated with deviation from what might be considered underlying 'normal' age-related change is key to fully understanding the life course epidemiology of these conditions and for identifying means of preventing future disease. 
thus important. We would hope that the evidence suggesting that the early midlife rate of change in $\mathrm{BP}$ - and possibly in other measures of cardiometabolic risk - is related to $\mathrm{CV}$ risk will have been thoroughly investigated and potential interventions robustly tested. The evidence that occupational cohorts have milder rises in BP suggests what is potentially modifiable, even in the western context. Rapid technological developments will mean that more detailed longitudinal phenotypes of cardiometabolic function and new measures of subclinical disease progression in large samples will become increasingly feasible and provide methods of capturing continual and dynamic changes in ways that do not put too much of a burden on study participants. This will enable investigation, for example, of the impact of short-term as well as longer-term variations in measures such as BP on disease development. Similarly, developments in the field of epigenetics have the potential to explain the biological mechanisms linking early life and later disease. The growing number of intercohort collaborations will continue in order to both provide the statistical power for analyses and the replication of findings in different populations. Such collaborations will also allow for the increasing application of methods for establishing causal associations.

Financial \& competing interests disclosure

$R$ Hardy and D Kuh are funded by the UK Medical Research Council (MC_UU_12019/2, MC_ $U U_{-}$12019/4). DA Lawlor works in a unit that receives funding from the UK Medical Research Council (MC_ UU_12013/5). The authors have no other relevant affiliations or financial involvement with any organization or entity with a financial interest in or financial conflict with the subject matter or materials discussed in the manuscript apart from those disclosed.

No writing assistance was utilized in the production of this manuscript.

\section{Open access}

This work is licensed under the Creative Commons Attribution 4.0 License. To view a copy of this license, visit http://creativecommons.org/licenses/by/4.0/

\section{References}

Papers of special note have been highlighted as:

- of interest; $\bullet$ of considerable interest

1 Strong JP, Malcom GT, McMahan CA et al. Prevalance and extent of atherosclerosis in adolescents and young adults: implications for prevention from the Pathobiological Determinants of Atherosclerosis in Youth Study. JAMA 281(8), 727-735 (1999).

2 Palinski W, Napoli C. The fetal origins of atheroscelrosis: maternal hypercholesterolemia and choleserol-lowering or antioxidant treatment during pregnancy influence in utero programming and postnatal susceptibility to atherogenesis. FASEB J. 16(11), 1348-1360 (2002).

3 Goff DC, Lloyd-Jones DM, Bennett G et al. 2013 ACC/AHA guideline on the assessment of cardiovascular risk: a report of the American College of Cardiology/American Heart Association Task Force on practice guidelines. Circulation 129(25 Suppl. 2), S49-S73 (2014).

JBS3 Board.. Joint British Societies' consensus recommendations for the prevention of cardiovascular disease (JBS3). Heart 100(Suppl. 2), ii1-ii67 (2014).

5 Expert Dyslipidemia Panel of the INternational Society Panel members. An International Atherosclerosis Society position paper: global recommendations for the management of dyslipidemia - full report. J. Clin. Lipidol. 8(1), 29-60 (2014).

6 Lloyd-Jones DM, Hong Y, Labarthe D et al. Defining and setting national goals for cardiovascular health promotion and disease reduction: the American Heart Association's strategic impact goal through 2020 and beyond. Circulation 121(4), 586-613 (2010).

7 Mishra GD, Ben-Shlomo Y, Kuh D. A life course approach to health behaviours: theory and methods. In: Handbook of Behavioural Medicine. Methods and Applications. Steptoe A (Ed.). Springer, NY, USA, 525-540 (2010).

8 Schooling M, Kuh D. A Life Course Appraoch to Women's Health. Kuh D, Hardy R (Eds). Oxford University Press, UK (2002).

9 A Life Course Approach to Chronic Disease Epidemiology: Tracing the Origins of Ill-Health from Early to Adult Life (2nd Edition). Kuh D, Ben-Shlomo Y (Eds). Oxford University Press, UK (2004).

10 Ben-Shlomo Y, Kuh D. A life course approach to chronic disease epidemiology: conceptual models, empirical challenges, and interdisciplinary perspectives. Int. J. Epidemiol. 31(2), 285-293 (2002).

- Original definitions and models for life course epidemiology are outlined.
11 Barker DJP. Mothers, Babies and Health in Later Life. (2nd Edition). Churchill Livingstone, UK (1998).

12 Kuh D, Smith GD. The life course and adult chronic disease: an historical perspective with particular reference to coronary heart disease. In: A Life Course Approach to Chronic Disease Epidemiology (2nd Edition). Kuh D, Ben-Shlomo Y (Eds). Oxford University Press, UK, 15-37 (2004).

13 Mishra GD, Nitsch D, Black S, De Stavola B, Kuh D, Hardy R. A structured approach to modelling the effects of binary exposure variables across the life course. Int. J. Epidemiol. 38(2), 528-537 (2009).

14 Hallqvist J, Lynch J, Bartley M, Lang T, Blane D. Can we disentangle life course processes of accumulation, critical period and social mobility? An analysis of disadvantaged socio-ecomomic positions and myocardial infarction in the Stockholm Heart Epidemiology Porgram. Soc. Sci. Med. 58(8), 1555-1562 (2004).

15 De Stavola B, Nitsch D, dos Santos Silva I et al. Statistical issues in life course epidemiology. Am. J. Epidemiol. 163(1), 84-96 (2006).

16 Wills A, Tilling K. Modelling repeat exposures: some examples from life course 
epidemiology. In: A Life Course Approach to Healthy Ageing. Kuh D, Cooper R, Hardy R, Richards M, Ben-Shlomo Y (Eds). Oxford University Press, UK, 91-108 (2014).

17 Smith GD. Epidemiology, epigenetics and the 'gloomy prospect': embracing randomness in population health research and practice. Int. J. Epidemiol. 40(3), 537-562 (2011).

18 Kuh D, Richards M, Cooper R, Hardy R, Ben-Shlomo Y. Life course epidemiology, ageing research and maturing cohort studies: a dynamic combination for understanding healthy ageing. In: A Life Course Approach to Healthy Ageing. Kuh D, Cooper R, Hardy R, Richards M, Ben-Shlomo Y (Eds). Oxford University Press, UK, 3-15 (2014).

19 Lawlor DA, Hardy R. A life course approach to cardiovascular and metabolic ageing. In: A Life Course Approach to Healthy Ageing. Kuh D, Cooper R, Hardy R, Richards M, Ben-Shlomo Y (Eds). Oxford University Press, UK, 146-161 (2014).

- Recent book chapter reviewing the evidence relating to cardiometabolic trajectories, their risk factors and how they relate to outcomes.

20 Smith GD, Ebrahim S. Mendelian randomization: prospects, potentials, and limitations. Int. J. Epidemiol. 33(1), 30-42 (2004).

21 Wills AK, Lawlor DA, Matthews F et al. Life course trajectories of systolic blood pressure using longitudinal data from UK cohorts. PLoS Med. 8(6), e1000440 (2011).

- Analysis of life course blood pressure trajectories using multiple longitudinal rather than cross-sectional cohort studies.

22 Pearson JD, Morrell CH, Brant IJ, Landis PK, Fleg JL. Age-associated changes in blood pressure in a clongitudinal study of healthy men and women. J. Gerontol. A Biol. Sci. Med. Sci. 52(3), M177-M183 (1997).

23 Franklin SS, Gustin W, Wong ND, Weber MA, Kannel WB, Levy D. Hemodynamic patterns of age-related changes in blood pressure. The Framingham Heart Study. Circulation 96(1), 308-315 (1997).

24 Labarthe DR, Dai S, Fulton JE, Harrist RB, Shah SM, Eissa MA. Systolic and fourt-and fifth-phase diastolic blood pressure from 8 to 18 years: Project HeartBeat! Am. J. Prev. Med. 37(1 Suppl.), S86-S89 (2009).

- Analysis of blood pressure trajectories in childhood in a longitudinal study with multiple repeated measures.

25 Reitz C, Luchsinger JA. Relation of blood pressure to cognitive impairment and dementia.
Curr. Hypertens. Rev. 3(3), 166-176 (2007).

26 Carvalho JJ, Baruzzi PF, Howard PF et al. Blood pressure in four remote populations in the INTSERSALT study. Hypertension 14(3), 238-246 (1989).

27 Gurven M, Blackwell AD, Rodriguez DE, Stieglitz J, Kaplan H. Does blood pressure inevitably rise with age? Longitudinal evidecne among forager-horticulturists. Hypertension 60(1), 25-33 (2012).

28 Wills AK, Lawlor DA, Muniz-Terrera G et al. Population heterogeneity in trajectories of midlife blood pressure: the 1946 British Birth Cohort Study. J. Hypertens. 28(4), 679-686 (2012).

- Study defining latent classes of blood pressure change in midlife and relating them to early life risk factors and angina.

29 Allen NB, Siddique J, Wilkins JT et al. Blood pressure trajectories in early adulthood and subclinical atherosclerosis in middle age. JAMA 311(5), 490-497 (2014).

-• Similar study relating latent classes of blood pressure to cardiovascular outcome.

30 Jolliffe CJ, Janssen I. Distribution of lipoproteins by age and gender in adolescents. Circulation 114(10), 1056-1062 (2006).

31 Dai S, Fulton JE, Harrist RB, Grunbaum JA, Steffen LM, Labarthe DR. Blood lipids in children: age-related patterns and association with body-fat indices: Project HeartBeat! Am. J. Prev. Med. 37(Suppl. 1), S56-S64 (2009).

32 Singh GM, Danaei G, Pelizzari PM et al. The age associations of blood pressure, cholesterol, and glucose: analysis of health examinations surveys from international populations. Circulation 125(18), 2204-2211 (2012).

33 Mann D, Reynolds K, Smith D, Muntner P. Terns in statin use and low-density lipoprotein cholesterol levels among US adults: impact of the 2001 National Cholesterol Education Program guidelines. Ann. Pharmacol. 42, 1208-1215 (2008).

34 Jolliffe CJ, Janssen I. Development of age-specific adolescent metabolic syndrome criteria that are linked to the Adult Treatment Panel III and International Diabetes Federation criteria. J. Am. Coll. Cardiol. 49(8), 891-898 (2007).

35 Shimokata H, Muller DC, Fleg JL, Sorkin J, Ziemba AW, Andres R. Age as an independent determinant of glucose tolerance. Diabetes 40(1), 44-51 (1991).

36 Reaven G. Age and glucose intolerance: effect of fitness and fatness. Diabetes Care 26(2), 539-540 (2003).
37 Sattar N, Greer IA. Pregnancy complications and maternal cardiovascular risk: opportunities for intervention and screening? BMJ 325(7356), 157-160 (2002).

38 Rich-Edwards J. Reproductive health as a sentinel of chronic disease in women. Womens Health (Lond. Engl.) 5(2), 101-105 (2009).

- Review of the evidence suggesting that women's reproductive health is a marker of chronic disease.

39 Lakshman R, Forouhi NG, Sharp SJ et al. Early age at menarche associated with cardiovascular disease and mortality. J. Clin. Endocrinol. Metabol. 94(12), 4953-4960 (2009).

40 Hardy R, Kuh D, Whincup PH, Wadsworth MEJ. Age at puberty and adult blood pressure and body size in a British Birth Cohort Study. J. Hypertens. 24(1), 59-66 (2006).

41 Kivimaki M, Lawlor DA, Smith GD et al. Association of age at menarche with cardiovascular risk factors, vascular structure, and function in adulthood: the Cardiovascular Risk in Young Finns study. Am. J. Clin. Nutr. 87(6), 1876-1882 (2008).

42 Pierce M, Kuh D, Hardy R. Role of lifetime body mass index in the association bewteen age at puberty and adult lipids: findings from men and women in a British birth cohort. Ann. Epidemiol. 20(9), 676-682 (2010).

43 Lawlor DA, Emberson JR, Ebrahim S et al. Is the association between parity and coronary heart disease due to biological effects of pregnancy or adverse lifestyle risk factors associated with child-rearing? Findings from the British Women's Heart and Health Study and the British Regional Heart Study. Circulation 107(9), 1260-1264 (2003).

44 Hardy R, Lawlor DA, Black S, Wadsworth MEJ, Kuh D. Number of children and cardiovascular risk factors at age 53 years in men and women. BJOG 114(6), 721-730 (2007).

45 Harskamp RE, Zeeman GG. Preeclampsia: at risk for remote cardiovascular disease. Am. J. Med. Sci. 334(4), 291-295 (2007).

46 Bellamy L, Casas J-P, Hingorani AD, Williams DJ. Pre-eclampsia and risk of cardiovascular disease and cancer in later life: systematic review and meta-analysis. $B M J$ 335(7627), 974 (2007).

47 Smith GD, Hyponnen E, Power C, Lawlor DA. Offspring birth weight and parental mortality: a prospective observational study and meta-analysis. Am. J. Epidemiol. 166(2), 160-169 (2007). 
48 Smith GC, Pell JP, Walsh D. Pregnancy complications and maternal risk of ischaemic heart disease. Lancet 357(9273), 2002-2006 (2001).

49 Catov JM, Wu CS, Olsen J, Sutton-Tyrrell K, Li J, Nohr EA. Early or recurrent preterm birth and maternal cardiovascular disease risk. Ann. Epidemiol. 20 (8), 604-609 (2010).

50 Lokkegaard E, Jovanovic Z, Heitmann BL, Keiding N, Ottesen B, Pedersen AT. The association between early menopause and risk of ischaemic heart disease: influence of hormone therapy. Maturitas 53(2), 226-233 (2006).

51 Matthews KA, Crawford SL, Chae CU et al. Are changes in cardiovascular disease risk factors in midlife women due to chronological aging or to the menopausal transition? J. Am. Coll. Cardiol. 54(25), 2366-2373 (2009).

52 Kok HS, van Asselt KM, vab der Schouw YT et al. Heart disease risk determines menopausal age rather than the reverse. J. Am. Coll. Cardiol. 47(10), 483-493 (2006).

53 Gast GC, Pop VJ, Samsioe GN et al. Vasomotor menopausal symptoms are associated with increased risk of coronary heart disease. Menopause 18(2), 146-151 (2011).

54 Thurston RC, Sutton-Tyrrell K, Everson-Rose SA, Hess R, Matthews KA. Hot flashes and subclinical cardiovascular disease: findings from the Study of Women's Health Across the Nation Heart Study. Circulation 118(12), 1234-1240 (2008).

55 Gast GC, Grobbee DE, Pop VJ et al. Menopausal complaints are associated with cardiovascular risk factors. Hypertension 51(6), 1492-1498 (2008).

56 Huxley R, Owen CG, Whincup PH et al. Is birth weight a risk factor for ischemic heart disease in later life? Am. J. Clin. Nutr. 85(5), 1250 (2007).

- Systematic review and meta-analysis summarizing evidence linking birth weight and coronary heart disease; one of a series of reviews summarizing evidence relating to the developmental origins of cardiovascular disease.

57 Whincup PH, Kaye SJ, Owen CG et al. Birth weight and risk of Type 2 diabetes: a systematic review. JAMA 300(24), 28862897 (2008).

58 Huxley R, Neil A, Collins R. Unravelling the fetal origins hypothesis: is there really an inverse association between birth weight and subsequent blood pressure? Lancet 360 (9334), 659-665 (2002).
59 Huxley R, Owen CG, Whincup PH, Cook DG, Colman S, Collins R. Birth weight and subsequent cholesterol levels: exploration of the 'fetal origins' hypothesis. JAMA 292(22), 2755-2764 (2004).

60 Law CM, de Swiet M, Osmond C et al. Initiation of hypertension in utero and its amplification throughout life. $B M J$ 306(6868), 24-27 (1993).

61 Koupilova I, Leon DA, Lithell HO, Berglund L. Size at birth and hypertension in longitudinally followed 50-70 year-old men. Blood Press. 6(4), 223-228 (1997).

62 Hardy R, Kuh D, Langenberg C, Wadsworth MEJ. Birthwight, childhood social class, and change in adult blood pressure in the 1946 British birth cohort. Lancet 362(9391), 1178-1183 (2003).

63 Uiterwaal CS, Anthony S, Launer LJ et al. Birth weight, growth, and blood pressure: an annual follow-up of children aged 5 through 21 years. Hypertension 30 (2 Pt 1), 267-271 (1997).

64 Williams S, Poulton R. Birth size, growth and blood pressure between ages of 7 and 26 years: failure to support the fetal origins hypotehsis. Am. J. Epidemiol. 155(9), 849-852 (2002).

65 Chen W, Srinivasan SR, Berensen GS. Amplification of the association between birthweight and blood pressure with age: the Bogalusa Heart Study. J. Hypertens. 28(10), 2046-2052 (2010).

66 Hardy R, Sovio U, King VJ et al. Birth weight and blood pressure in five European birth cohorts studies: an investigation of confounding factors. Eur. J. Pub. Health 16(1), 21-30 (2006).

67 Inskip HM, Godfrey KM, Robinson SM et al.; SWS Study Group. Cohort profile: the Southampton Women's Survey. Int. J. Epidemiol. 35(1), 42-48 (2006).

68 Cooper R, Atherton K, Power C. Gestational age and risk factors for cardiovascular disease: evidence from the 1958 British birth cohort followed to mid-life. Int. J. Epidemiol. 38(1), 235-244 (2008).

69 Keag O, Stock S, Norman J. Long-term childhood outcomes following caesarean section: systematic review and meta-analysis. Arch. Dis. Child. Fetal Neonatal Ed. 99(Suppl. 1), A8-A9 (2014).

70 Yeung EH, Druschel C. Cardiometabolic health of children conceived by assisted reproductive technologies. Fertil. Steril. 99(2), 318-326 (2013).

71 Park MH, Falconer C, Viner RM, Kinra S. The impact of childhood obesity on morbidity and mortality in adulthood: a systematic review. Obes. Rev. 13(11), 985-1000 (2012).

72 Owen CG, Whincup PH, Orfei L et al. Is body mass index before middle age related to coronary heart disease risk in later life? Evidence from observational studies. Int. J. Obes. (Lond.) 33(8), 866-877 (2009).

73 Baker JL, Olsen LW, Sorensen TIA. Childhood body-mass index and the risk of coronary heart disease in adulthood. $N$. Engl. J. Med. 357(23), 2329-2337 (2007).

74 Barker DJ, Osmond C, Forsen T, Kajantie E, Eriksson JG. Trajectories of growth among children who have coronary events as adults. N. Engl. J. Med. 353(17), 1802-1809 (2005).

75 Eriksson JG. Early growth and coronary heart disease and Type 2 diabetes: findings from the Helsinki Birth Cohort Study (HBCS). Am. J. Clin. Nutr. 94 (6 Suppl.), 1799S-1802S (2011).

76 Must A, Jacques PF, Dallal GE, Bajema CJ, Dietz WH. Long-term morbidity and mortality of overweight adolescents. A follow-up of the Harvard Growth Study of 1922 to 1953. N. Engl. J. Med. 327(19), 1350-1355 (1992).

77 Lawlor DA, Benfield L, Logue J et al. Association between general and central adiposity in childhood, and change in these, with cardiovascular risk factors in adoleescence: prospective cohort study. BMJ 340, c6224 (2010).

78 Howe LD, Tilling K, Benfield L et al. Changes in ponderal index and body mass index across childhood and their associations with fat mass and cardiovascular risk factors at age 15. PLoS ONE 5(12), e15186 (2010).

79 Wills AK, Hardy RJ, Black S, Kuh DJ. Trajectories of overweight and body mass index in adulthood and blood pressure at age 53: the 1946 British Birth Cohort Study. J. Hypertens. 28(4), 679-686 (2010).

80 Juonala M, Magnussen CG, Berensen GS et al. Childhood adiposity, adult adiposity and cardiovascular risk factors. $N$. Engl. J. Med. 365(20), 1876-1885 (2011).

-• One of the few studies to investigate whether childhood adiposity is associated with adult cardiovascular risk independent of adult adiposity.

81 Kivimaki M, Smith GD, Timpson NJ et al. Lifetime body mass index and later atherosclerosis risk in young adults: examining causal links using mendelian randomization in the Cardiovascular Risk in Young Finns study. Eur. Heart J. 29(20), 2552-2560 (2008). 
82 Nordestgaard BG, Palmer TM, Benn M et al. The effect of elevated body mass index on ischemic heart disease risk: causal estimates from a Mendelian randomisation approach. PLoS Med. 9(5), e100121 (2012).

83 Paajanen TA, Oksala NK, Kuukasjarvi P, Karhunen PJ. Short stature is associated with coronary heart disease: a systematic review of the literature and meta-analysis. Eur. Heart J. 31(14), 1802-1809 (2010).

84 Wadsworth MEJ, Hardy R, Paul AA, Marshall SF, Cole TJ. Leg and trunk length at 43 years in relation to childhood health, diet and family circumstances; evidence from the 1946 national birth cohort. Int. J. Epidemiol. 31(2), 383-390 (2002).

85 Whitley E, Gunnell D, Smith GD, Holly JM, Martin RM. Childhood circumstances and anthropometry: the Boyd Orr cohort. Ann. Hum. Biol. 35(5), 518-534 (2008).

86 Langenberg C, Hardy R, Kuh D, Wadsworth M. Influence of height, leg length and trunk length on pulse pressure. J. Hypertens. 21(3), 537-543 (2003).

87 Ferrie JE, Langenberg C, Shipley MJ, Marmot MG. Birth weight, components of height and coronary heart disease: evidence from the Whitehall II study. Int. J. Epidemiol. 35(6), 1532-1542 (2006).

88 Skidmore PML, Hardy RJ, Kuh DJ, Langenberg C, Wadsworth MEJ. LIfe course body size and lipid levels at 53 years in a British birth cohort. J. Epidemiol. Community Health 61(3), 215-220 (2007).

89 Lawlor DA, Ebrahim S, Smith GD. The associations between components of adult height and Type II diabetes and insulin resistance: British Women's Heart Study. Diabetologia 45(8), 1097-1106 (2002).

90 Gunnell D, Whitley E, Upton M, McConnachie A, Smith GD, Watt G. Associations of height, leg length, and lung function with cardiovascular risk factors in the Midspan Family Study. J. Epidemiol. Community Health 57(2), 141-146 (2003).

91 Langenberg C, Hardy R, Breeze E, Kuh D, Wadsworth MEJ. Influence of short stature on the change in pulse pressure, systolic and diastolic blood pressure from age 36 to 53 years: an analysis using multilevel models. Int. J. Epidemiol. 34(4), 905-913 (2005).

92 Johnson W, Kuh D, Tikhonoff V et al. Body mass index and height from infancy to adulthood and carotid intima-media thickness at 60 to 64 years in the 1946 British Birth Cohort Study. Arterioscler. Thromb. Vasc. Biol. 34, 654-660 (2014).

93 Galobardes B, Smith GD, Lynch JW. Systematic review of the influence of childhood socioeconomic circumstances on risk of cardiovascular disease in adulthood. Ann. Epidemiol. 16(2), 91-104 (2006).

94 Galobardes B, Lynch JW, Smith GD. Childhood socioeocnomic circumstances and cause-specific mortality in adulthood: systematic review and interpretation. Epidemiol. Rev. 26, 7-21 (2004).

95 Galobardes B, Lynch JW, Smith GD. Is the association between childhood socioeconomic circumstances and cause-specific mortality established? Update of a systematic review. J. Epidemiol. Community Health 62(5), 387-390 (2008).

- Most recent of a series of systematic reviews linking childhood socioeconomic position with coronary heart disease, showing the relationship holds in women and in more contemporary cohorts in which overall childhood living conditions have improved.

96 Colhoun H, Hemingway H, Poulter N. Socio-economic status and blood pressure: an overview analysis. J. Hum. Hypertens. 12(2), 91-110 (1998).

97 Batty GD, Leon DA. Socio-economic position and coronary heart disease risk factors in children and young people. Evidence from the UK epidemiological studies. Eur. J. Pub. Health 12(4), 263-272 (2002).

98 Lawlor DA, Smith GD. Early life determinants of adult blood pressure. Curr. Opin. Nephrol. Hypertens. 14(3), 259-264 (2005).

99 Lawlor DA, Ebrahim S, Smith GD; British Women's Heart and Health Study. Socioeconomic position in childhood and adulthood and insulin resistance: cross sectional survey using data from British Women's Heart and Health Study. BMJ 325(7368), 805 (2002).

100 Lawlor DA, Smith GD, Ebrahim S. Association between childhood socioeconomic status and coronary heart disease risk among postmenopausal women: findings from the British Women's Heart and Health Study. Am. J. Pub. Health. 94(8), 1386-1392 (2004).

101 Murray ET, Mishra GD, Kuh D, Guralnik J, Black S, Hardy R. Life course models of socioeconomic position and cardiovascular risk factors: 1946 birth cohort. Ann. Epidemiol. 21(8), 589-597 (2011).

102 Langenberg C, Kuh D, Wadsworth MEJ, Brunner E, Hardy R. Social circumstances and education: life course origins of social inequalities in metabolic risk in a prospective national birth cohort. Am. J. Pub. Health. 96(12), 2216-2221 (2006).
103 McCarron P, Smith GD, Okasha M, McEwen $\mathrm{J}$. Blood pressure in young adulthood and mortality from cardiovascular disease. Lancet 355(9213), 1430-1431 (2000).

104 Sundstrom J, Neovius M, Tynelius P, Rasmussen F. Association of blood pressure in late adolescence with subsequent mortality: cohort study of Swedish male conscripts. BMJ 342, d643 (2011).

105 Raitakari OT, Jounala M, Kahonen M et al. Cardiovascular risk factors in childhood and carotid artery intima-media thickness in adulthood: the Cardiovascular Risk in Young Finns study. JAMA 290(17), 2277-2283 (2003).

106 Juonala M, Magnussen CG, Venn A et al. Influence of age on associations between childhood risk factors and carotid intimamedia thickness in adulthood: the Cardiovascular Risk in Young Finns study, the Childhood Determinats of Adult Health study, the Bogalusa Heart Study, and the Muscatine Study for the International Childhood Cardiovascular Cohort (i3C) Consortium. Circulation 122(24), 1604-1611 (2010).

-• Investigation of cardiovascular risk factors at various ages in childhood in relation to carotid intima-media thickness in adulthood.

107 Li X, Li S, Ulusoy E, Chen W, Srinivasan SR, Berensen GS. Childhood adiposity as a predictor of cardiac mass in adulthood: the Bogalusa Heart Study. Circulation 110 (22), 3488-3492 (2004).

108 Ference BA, Julius S, Mahajan N, Levy PD, Williams KA, Flack JM. Clinical effect of naturally random allocation to lower systolic blood pressire beginning before the development of hypertension. Hypertension 63(6), 1182-1188 (2014).

109 Allen N, Berry JD, Ning H, Van Horn L, Dyer A, Lloyd-Jones DM. Impact of blood pressure change during middle age on the remaining lifetime risk for cardiovascular disease: the Cardiovascular Lifetime Risk Pooling Project. Circulation 125(1), 37-44 (2012).

-• Study illustrating how change in blood pressure impacts on the lifetime risk for cardiovascular disease.

110 Ghosh AK, Hardy RJ, Francis DP et al. Midlife blood pressure change and left ventricular mass and remodelling in older age in the 1946 British Birth Cohort Study. Eur. Heart J. doi:10.1093/eurheartj/ehu389 (2014) (Epub ahead of print). 
